\title{
An Intelligent Agent for Supporting User Navigation in a Hyperspace
}

\author{
A. Micarelli and F. Sciarrone \\ Dipartimento di Discipline Scientifiche - Sez. Informatica \\ Università di Roma 3 \\ Via della Vasca Navale 84, 00146 Roma, Italy \\ E-mail: micarel@inf.uniroma3.it
}

\begin{abstract}
In this paper we describe an approach for adaptive hypermedia navigation, based on the use of an intelligent agent which tries to automatically infer user learning goals during the navigation. Our proposal takes inspiration from Case-Based Reasoning. The indexing problem, critical in any Case-Based Reasoner, has been solved by transforming that problem to a pattern recognition problem, and by using an artificial neural network, well suited for the task of pattern recognition.
\end{abstract}

\section{Keywords}

Navigational Aids, Intelligent Agents, Case-Based Reasoning, Artificial Neural Networks.

\section{INTRODUCTION}

In this work we present an approach for the realization of hypermedia/multimedia systems that allow for adaptive navigation in the hyperspace, particularly suited for educational purposes.

Generally speacking, a distinguished feature of the "Intelligent Systems" is the capability of adapting their behavior to the needs and characteristics of the particular user. Examples of such systems are the so-called Intelligent Tutoring Systems (ITS), i.e., expert systems that have been conceived for educational applications (Wenger, 1987). They are characterized by the computational ability to interpret the student's problem solving behavior, building up through the interaction a qualitative representation of the learning status of the student, called student model. This ability provides highly adaptive orientation to the learning session. 
Hypermedia systems can be viewed as tools that keep a great storage of different kinds of information residing in various media (text, graphics, animations, still images, video, sounds), and permit quick access to such information (Nielsen, 1990). They are information management tools that are not per se pedagogical, but can conveniently be used as learning systems, in the sense that they can support exploratory type of learning. However, as the size of the hypermedia data base grows, unsupported navigation often causes the users to get lost in the hyperspace. Therefore, adequate aids for an adaptive navigation through this space are needed (Dillon et al, 1990). Hypermedia systems are different from ITSs, mainly because of the different treatment of information either as display data (which is non-computational) or as knowledge (which is computational). For this reason the task of building a qualitative student model (intended as a knowledge base on the learning status of the student) while the student navigates in a hypermedia system is problematic. It is reasonably easy to keep trace of the navigation performed by the student. There are some more problems for its interpretation: in fact, we believe that the student modeling activity is reliable only if what is followed and monitored by the system is the problem solving behavior of the student. In the rest of the paper we describe our proposed solution for adaptive knowledge navigation, based on the use of an intelligent agent (Rieken, 1994) capable of inferring user learning goals during the navigation.

\section{AN INTELLIGENT AGENT FOR KNOWLEDGE NAVIGATION}

In order to define a suitable approach for adaptive knowledge navigation, we have chosen some existing hypermedia systems and interviewed and observed expert tutors during the interaction with students who were navigating in the hyperspace. One of the application domains we have chosen is Rome in the Middle Ages. This hypermedia system has been developed on a IBM MS-DOS platform (@ Garamond). Seven thematic walking (thematic paths) in Rome (with the associated learning goals) are possible in order to learn about ancient buildings, such as churchs and towers, belonging to the Middle Age era. Every node is composed of some photos and historical notes about the ancient buildings. The structure of the hypermedia is based on a direct graph composed of 46 nodes and 70 links. At the beginning of a session the tutor has given complete initiative to the students who have been allowed to freely navigate in the hyperspace. The tutor has monitored the students navigation, and has given suggestions only when a help has been explicitly requested (when a student tended to get lost in the hyperspace). In these cases the tutor, on the basis of the partial user/student path, has always been able to recognize the user/student intentions by determining the thematic path (and the associated learning goal) that closely matched with the partial user/student path and by assuming that the determined goal and path were the target of the student. With this assumption, he has given the right 
suggestions to the students, bringing them back on track if necessary. The inexact matching between "user path" and "prestored thematic paths" has been the core problem solving model of the tutor.

We have designed an intelligent agent for guided hypermedia navigation whose software architecture has been inspired by the above experiments with tutor and students. Our approach uses the Case-Based Reasoning (Kolodner, 1993) paradigm. Case-Based Reasoning (CBR) is an approach to problem solving and learning based on the retrieval and adaptation of old experience, embedded in a data base of "cases". It can be viewed as a five-step problem-solving process (Aamodt, 1994; Allen, 1994): 1. presentation of a new problem to be solved; 2. retrieval, from the case library, of the old case (and the associated solution) that closely matches the new problem; 3 . adaptation of the old solution to the new problem; 4. validation of the solution determined in the previous step; 5. update of the case library with the new solved case for use in future problem solving.

Reasoning by using past cases is a very frequent way to solve problems for humans. We have assumed that the previously described tutor behavior during the sessions with students can be modeled by using the CBR paradigm. In fact, the thematic paths (and the associated learning goals) can be viewed as "prestored cases" and the determination of the user intentions (i.e. the determination of the presumed goal of the user starting from the partial user path) can be viewed as the "new problem" of a Case-Based reasoner. When the student asks for help during the navigation, the system takes into consideration the partial student path and tries to find the nearest predefined didactic path in the case library (retrieve phase). The case retrieved from the case library, with the associated didactic goal, can then be used to help the user find the desired path (adapt phase), bringing her back on track if it is the case, in the style of model-tracing Intelligent Tutoring Systems (see Micarelli and Sciarrone, 1996 for a description of the main features of the proposed case-based architecture).

The greatest problem in automatizing this procedure lies in the resolution of the "indexing problem", i.e., in the determination of the prestored case in the case library that corresponds to the user learning goal. With our approach, the "indexing problem" has been reduced to the task of recognizing instances of patterns. In fact, the working hypothesis we have done is that what is needed from the partial user path is the set of nodes present in the path, regardless of their links, the time the user spent browsing each node and the last visited node. In this way the user behaviour can be represented as an "instance of pattern", constituted by a record of attributes relative to the partial user path (array whose i-th element contains the time spent on the i-th node plus an element relative to the last visited node). 


\section{THE INDEXING MODULE}

For the solution of this problem we have chosen a sub-symbolic approach, consisting in the use of an artificial neural network engine based on a three-layer perceptron (McClelland \& Rumelhart, 1986), well suited for pattern recognition. When a pattern (corresponding to the user behaviour) is presented as input to the network, the network computes an index array that corresponds to a rank ordered list of cases present in the case library. For the realization of the network a MultiLayer Perceptron with three distinct layers ha been used. The network has been implemented in $\mathrm{C}$ language. In Figure 1 an example of the feedforward structure is shown.

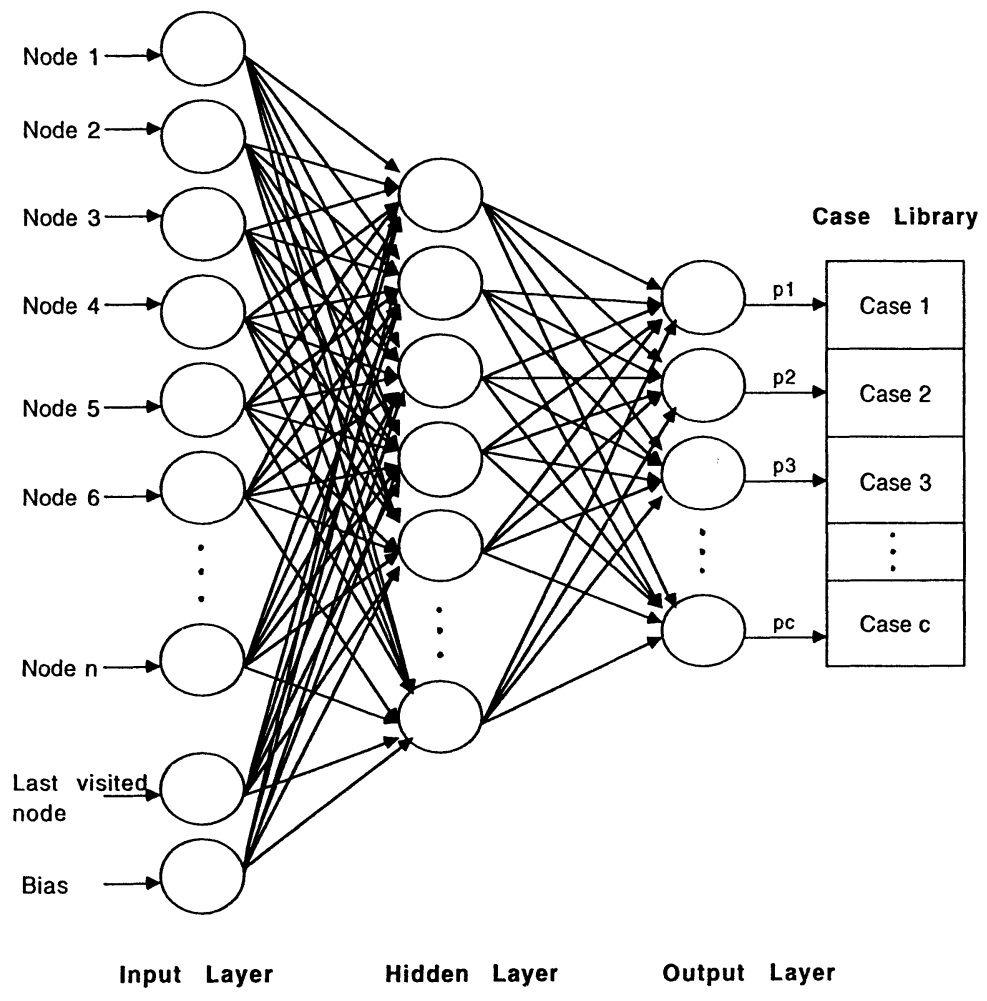

Figure 1 The feedforward structure of the perceptron. 
The first layer, the input layer, is composed of the neurons representing the user path, the last visited node and the bias value. The number of neurons which compose the output layer, corresponds to the number of cases in the case library. As for the hidden nodes, its number has been identified by means of experiments during the learning phase. The learning phase has been carried out in a supervised way and the Back-Propagation algorithm has been used. We have tested the network for the graph relative to an hypermedia system on "the neo-realism in the Italian cinema", which is under development in our University. In the final version, the hypermedia will be constituted by 66 nodes, 566 links, 150 screens, 80 still images, 10 video clips and 14 thematic paths. The training-set (i.e., the set of input-output right patterns used in the learning phase) relative to this hypermedia have been defined by the tutor. The network has learnt by iterations (epochs) by building the weights between it's neurons. The weight adjustment followed the rule:

$$
\omega_{i j}^{r+1}=\omega_{i j}^{r}+\eta \delta_{i} x_{i}+\alpha\left(\omega_{i j}{ }^{r}-\omega_{i j}^{r-1}\right)
$$

where $\omega_{i j}{ }^{r+1}$ represents the synaptic weight beetwen the $\mathrm{i}$-th and $\mathrm{j}$-th neuron calculated at the $(\mathrm{r}+1)$-th iteration, $h$ the learning rate (in the range $[0,1]), d_{i}$ the error relative to the neuron $i$ between the calculated output and the true output, $x_{i}$ the output of the i-th neuron, $a$ the momentum factor (in the range $[0,1]$ ). According to (McClelland and Rumelhart, 1986), we have used $\eta=0.5$ and $\alpha=0.9$. Besides, the starting values of the weights belonged to the range $[-0.3,+0.3]$. The following sigmoid (or S-shaped) function has been used as a transfer function:

$$
o_{j}=\left[1+\exp \left(\sum_{i} \omega_{i j} o_{i}\right)\right]^{-1}
$$

where $o_{j}$ represents the output of the j-th neuron, while $o_{i}$ is the output of the $\mathrm{i}$-th neuron connected to the input of the $\mathrm{j}$-th neuron.

The authoring process (see Figure 2) has been divided into the following steps:

1. Construction of a data base of training records. A training record is an input-output pair, where the output is the index array relative to the canonical paths present in the case library. The most suited case for the user is the associated one with the first in the descendent -ordered index array.

2. The test range process. The goal of this phase is to train the network presenting as input some of the already planned record sets.

3. The predict range process. In this phase, the author gives the network some inputs for which the output is already known in order to see the forecasting behaviour. 


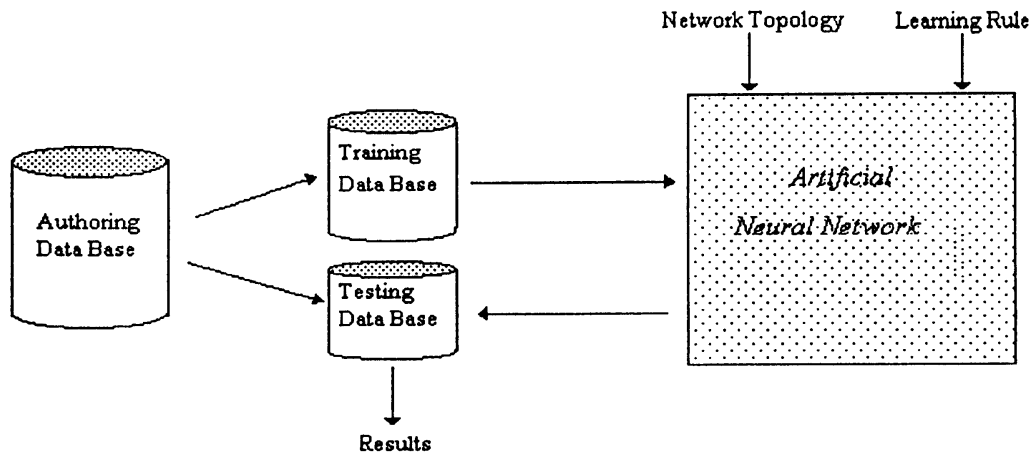

Figure 2 The Authoring Process.

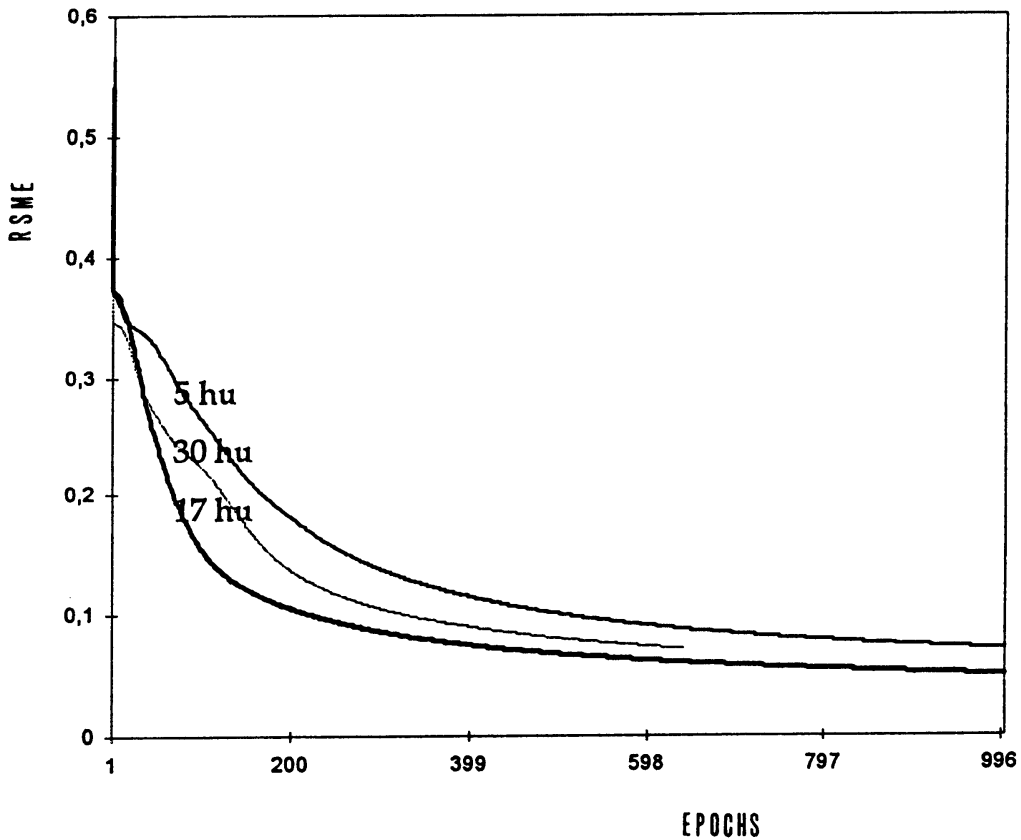

Figure 3 The Error Curves in the Learning Phase. 
We have tested several configurations of the network and determined the corresponding error curves. An error curve shows the network throughput during it's supervised learning activity. The error is defined by the mean-square difference between the right output vector (defined in the treining set) and the output vector generated by the network. Figure 3 shows the error curve for three different numbers of hidden nodes. We have chosen the configuration with 17 hidden units (hu) since it has been the fastest for the error convergence to zero.

Figure 4 shows the performance of the network as a function of the epochs and of the number of hidden nodes for a given number of training records. The configuration with 17 hidden nodes has reached a satisfactory correctness level (85\% performance in the caseof 1000 training record used in the learning phase).

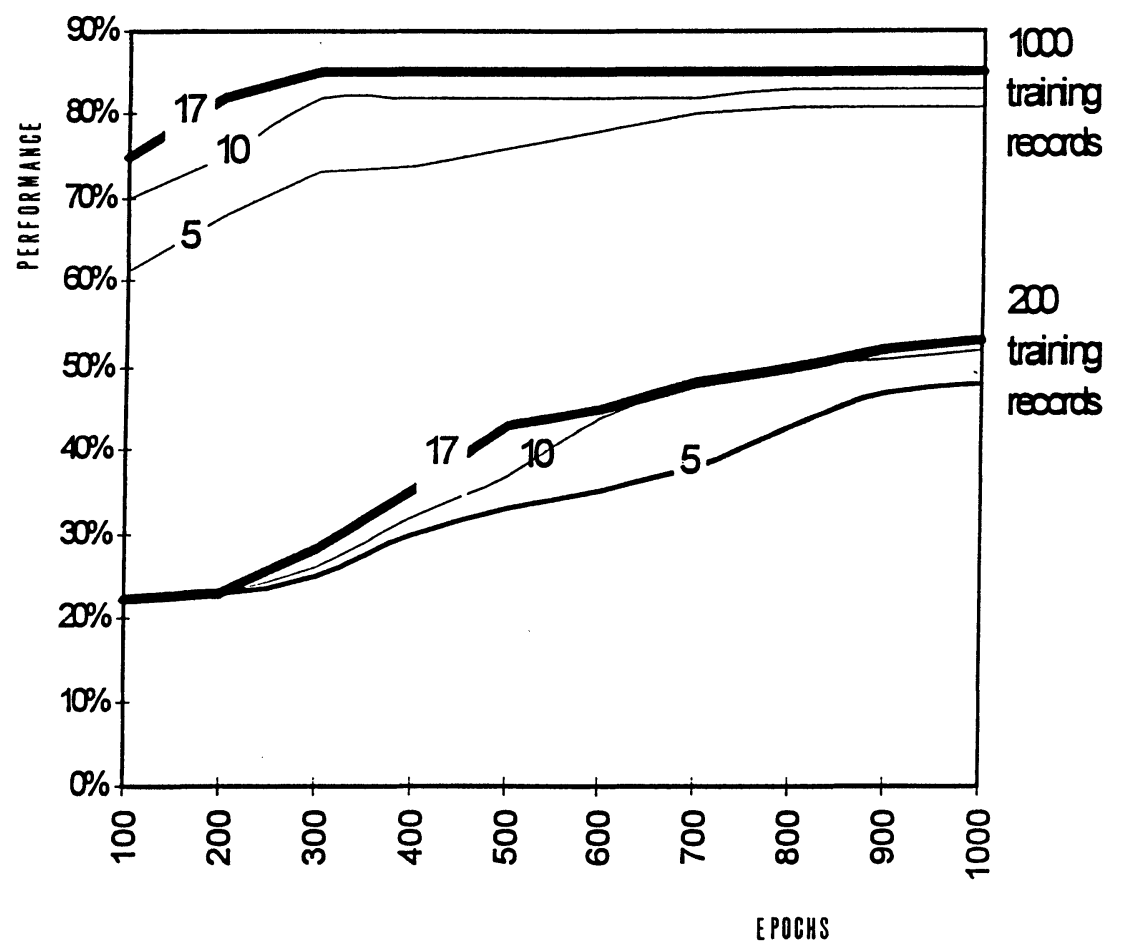

Figure 4 The Performance Curves. 


\section{CONCLUSIONS}

In this paper we have proposed a solution to the problem of adaptive knowledge navigation in a hyperspace. It consist in the use of an intelligent agent which tries to automatically infer user learning goals to guide the navigation. The problem of competence of the agent has been solved by means of a hybrid architecture, which takes inspiration from Case-Based Reasoning. The indexing problem, critical in any Case-Based Reasoner, has been solved by transforming that problem to a pattern recognition problem, and by using an artificial neural network, well suited for the task of pattern recognition. As for the performance of the network, the first tests have been encouraging. An extensive use of the system is being planned.

\section{REFERENCES}

Aamodt, A. and Plaza, E. (1994). "Case-Based Reasoning: Foundational Issues, Methodological Variations, and System Approaches". AI Communications, 7(1), 39-59.

Allen, B.P. (1994). "Case-Based Reasoning: Business Applications". Communications of the ACM, 37(3), 40-42.

Dillon, A., McKnight, C. and Richardson, J. (1990). "Navigation in Hypertext: A Critical Review of the Concept". In Interact '90 (eds. D. Diaper, D. Gilmore, G. Cockton and B. Shackel), Amsterdam:North Holland.

Kolodner, J. (1993). Case-Based Reasoning. San Mateo, Calif., Morgan Kaufmann.

McClelland, J.L. and Rumelhart, D.E. (1986). Parallel Distributed Processing, Explorations in the Microstructure of Cognition, Vol. 1: Foundations. MIT Press, Cambridge, MA.

Micarelli, A. and Sciarrone, F. (1996). "A Case-Based Toolbox for Guided Hypermedia Navigation". In proceedings of the Fifth International Conference on User Modeling UM96, Hawaii, January 2-5, pp. 129-136.

Nielsen, J. (1990). Hypertext and Hypermedia. San Diego, Calif., Academic Press.

Rieken, D. (1994). Special Issue on Intelligent Agents. Communications of the ACM, 37(7).

Wenger, E. (1987). Artificial Intelligence and Tutoring Systems. Los Altos, CA: Morgan Kaufmann. 